\title{
Deformation behaviour of a polycarbonate plate with a circular hole: finite elements model and experimental observations
}

\author{
Hung-Jue Sue and Albert F. Yee* \\ Macromolecular Research Center and Department of Materials Science and Engineering, \\ University of Michigan, Ann Arbor, Michigan 48109, USA \\ (Received 16 November 1987; accepted 3 February 1988)
}

\begin{abstract}
The unique and complex deformation behaviour of ductile polymers has received considerable attention in the polymer field. Much research has been conducted to study the yielding and post-yielding behaviour of polymers. In the case of two-phase polymer alloy systems, however, the deformation behaviour near the interface between the two phases has rarely been studied. The present paper describes the use of twodimensional (2-D) finite element methods (FEM) to simulate the deformation behaviour of a polycarbonate plate with a circular hole as a special case of two-phase polymer alloys. The FEM model involves consideration of the non-linear behaviour of the material. The FEM simulation results reveal that the maximum octahedral shear stress $\left(\tau_{o c t}\right)$ shifts from the equatorial region toward the $45^{\circ}$ region at the interface between the hole and the ductile matrix phase. How far the maximum $\tau_{\text {oct }}$ shifts along the interface depends on the stress concentration near the interface and the strain increment between the yield and the strain hardening regions. The shift in maximum $\tau_{\text {oct }}$ is due to the yield and strain softening character of ductile polymers. These results are verified by polycarbonate/hole (PC/hole) experiments.
\end{abstract}

(Keywords: polymer alloy; octahedral shear stress; yielding; post-yielding; non-linear stress-strain curve; FEM)

\section{INTRODUCTION}

Recent developments in toughened multi-phase polymer alloys ${ }^{1-4}$ emphasize the importance of understanding the large deformation behaviour of polymeric materials. For single phase polymers, there have been some efforts to probe, either theoretically, experimentally or numerically, their yielding ${ }^{5-10}$ and post-yielding ${ }^{10-13}$ behaviour. However, it is still not clear, from a continuum mechanics point of view, how the polymer becomes microscopically deformed. It is only understood that shear banding of a deformed specimen is a consequence of a strain softening phenomenon ${ }^{6}$. Moreover, in the case of multi-phase polymer alloys consisting of a dispersion of polymer particles embedded in a continuous matrix, not only is it not known how the matrix polymer locally deforms beyond the linear elastic regime near the interface, but also it is not known how strain softening affects the material surrounding the yielded material. These problems can be addressed using a continuum mechanics approach.

In 1933, Goodier ${ }^{14}$ derived the first classical linear elastic closed-form solutions for studying the stress-strain $(\sigma-\varepsilon)$ field around a spherical or cylindrical inclusion embedded in a continuous medium. Others ${ }^{15-18}$ have then either modified his solution or derived more versatile closed-form solutions for heterogeneous systems. For polymer alloys, closed-form solutions are extremely complex; they can be profitably studied using finite element method (FEM) simulations ${ }^{5,19-22}$. Most of the FEM simulations described in the literature use the assumption of linear elastic material properties. In practice, however, because of the stress concentration

\footnotetext{
* To whom correspondence should be addressed
}

effect due to the inclusion phase, the material at the interface may undergo local large scale plastic deformation while the bulk of the continuous matrix undergoes linear elastic deformation. In order to obtain a more accurate understanding of the deformation behaviour of multi-phase alloys, the non-linear material properties of the polymers must be considered.

A 2-D FEM model was constructed. A cylindrical inclusion (or hole), embedded in the polymer matrix, was used as a model stress concentrator. One interesting and striking result that emerged from the simulation is that with a more compliant inclusion (with respect to the matrix) embedded in a shear-yielding polymer matrix, when the matrix is loaded in tension, there is a shift of the maximum octahedral shear stress $\tau_{\text {oct }}$ around the inclusion (which can be a hole) from the equatorial region of the inclusion toward the $\theta=45^{\circ}$ region as the applied tensile stress is increased. Accordingly, the yielded zone formed initially at the equatorial region gradually extends toward the $\theta=45^{\circ}$ interface.

Polycarbonate tensile specimens, into which had been drilled $\mathrm{a} \frac{1}{16}{ }^{\prime \prime}(1.59 \mathrm{~mm})$ cylindrical hole, were tested and investigated under a stereomicroscope. The 2-D FEM simulation appears to correlate well with the experimental work.

\section{MODELLING USING FEM}

The MSC/NASTRAN ${ }^{\circledR}$ FEM package (64A, 1985 version) was employed to carry out a 2-D simulation. The advantage of using the MSC/NASTRAN ${ }^{\circledR}$ package is two-fold: it is capable of modelling typical polymer strain softening and hardening behaviours, and it can perform relatively large strain calculations.

In the present model, the following assumptions are 


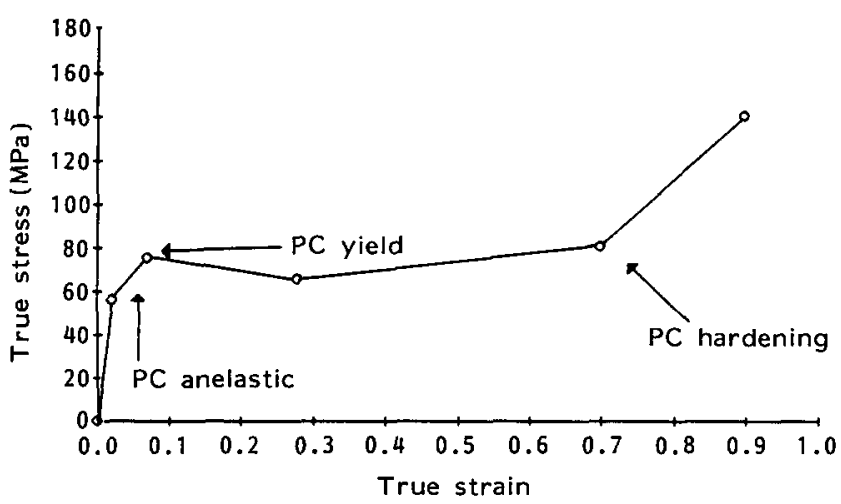

Figure 1 Typical piecewise linear stress-strain curve used for FEM simulations

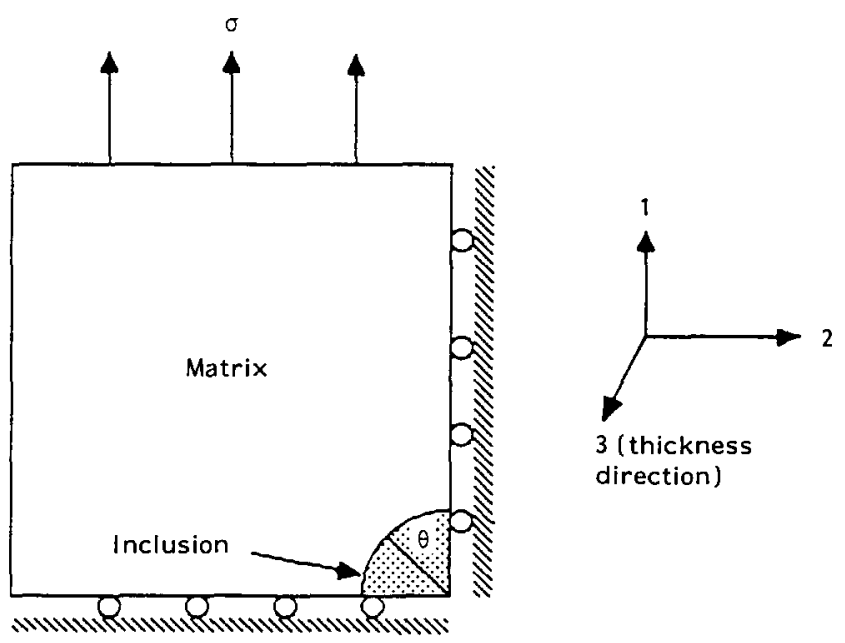

Figure 2 Schematic drawing of the FEM model. $\theta$ is defined as the angle between the tensile direction and the direction from the centre of the inclusion to the location of the maximum $\tau_{\text {oct }}$

made:

isotropy;

cylindrical dispersed phase with the cylinder axis perpendicular to the plane of the model;

perfect adhesion between the phases;

no interaction between dispersed inclusions;

piecewise linear stress-strain curve (Figure 1);

negligible thermal stress at the interface.

A far-field tensile stress is applied. The model is examined with or without lateral constraint. In addition, the Von Mises yield criterion was used. Furthermore, in the third direction, either a thin $\left(\sigma_{3}=0\right)$ or a thick $\left(\varepsilon_{3}=0\right)$ plate can be assumed (Figure 2). In the case of the thick plate condition, by assuming $\sigma_{3}=v\left(\sigma_{1}+\sigma_{2}\right)$ (where $v$ is Poisson's ratio) for the entire deformation process, it was found that the results closely parallel those of the thin plate. These findings can be verified by experiments on thin and thick plates of $\mathrm{PC} /$ hole specimens.

Owing to the rather expensive and time-consuming computation procedure, a simple FEM model mesh is generated (Figure 3) using the PATRAN ${ }^{\circledR}$ preprocessor to facilitate the simulation work. It is found that the simple model mesh sufficiently serves our purpose.

The deformation of many material pairs were simulated in these computer experiments. The polycarbonate/hole ( $\mathrm{PC} /$ hole) system is used to demonstrate the phenomenon of the shift of the maximum $\tau_{\text {oct }}$.

\section{EXPERIMENTAL}

Specimens of PC (Lexan) sheets $\left(\frac{1}{4}\right.$ and $\frac{1}{16}^{\prime \prime}, 6.35$ and $1.59 \mathrm{~mm}$, respectively) were cut and polished to dimensions of $7 \times 1 \times 1^{\prime \prime}(177.8 \times 25.4 \times 6.35 \mathrm{~mm})$ and $7 \times 1 \times \frac{1}{16}{ }^{\prime \prime}(177.8 \times 25.4 \times 1.59 \mathrm{~mm})$. No thermal treatment of the as-received material was performed. A cylindrical hole $\left(\frac{1}{16}{ }^{\prime \prime}, 1.59 \mathrm{~mm}\right)$ was then carefully drilled into the centre. Before conducting the tensile tests, the specimens were checked between crossed-polarizers in the microscope to see if residual stresses were present. None were found.

The PC specimen was mounted onto a screw-driven Instron testing machine using a pair of serrated face grips to hold both ends of the specimen. A crosshead speed of $0.05^{\prime \prime} \min ^{-1}\left(0.13 \mathrm{~mm} \mathrm{~min}^{-1}\right)$ was used in the test. A Nikon SMZ-2T stereomicroscope was used to observe the deformation behaviour of the PC/hole system. While the specimen was being tested in tension, a series of micrographs were obtained at stages wherein the shear bands began to grow. The temperature during the test was $25^{\circ} \mathrm{C}$

\section{RESULTS AND DISCUSSIONS}

In the FEM simulation, the load is increased in small increments. The finer the load increment used, the more precise the solution. At the same time, however, more computer time is needed to obtain the solutions. Consequently, only 18 load steps are used for the simulation work. Uniaxial tensile stress is applied to the model.

The FEM results of the $\mathrm{PC} /$ hole case are illustrated using the $\tau_{\text {oct }}$ contour plots (Figure 4). (Since the outcome of the thick and thin plate conditions are qualitatively similar, Figure 4 can be used to describe both the plane strain and plane stress cases.) Each contour curve in the plot is numbered to correspond to a stress level. A higher number indicates a higher stress state in the

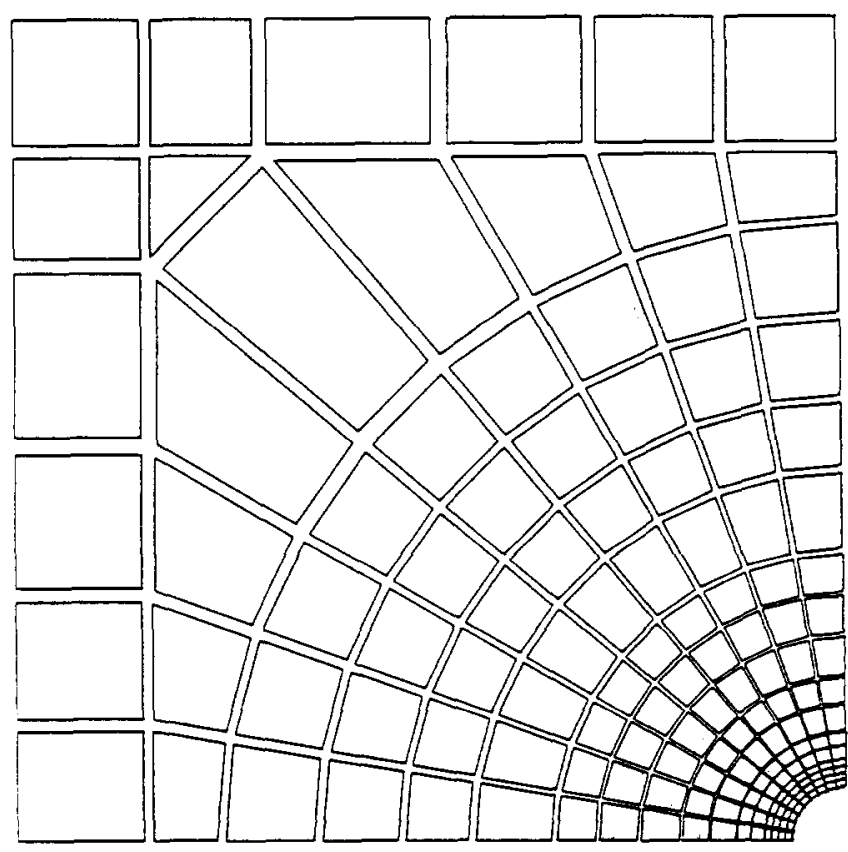

Figure 3 FEM model mesh generated using the :PATRAN ${ }^{\circledR}$ preprocessor. This simplified mesh is sufficient for the present work 
a

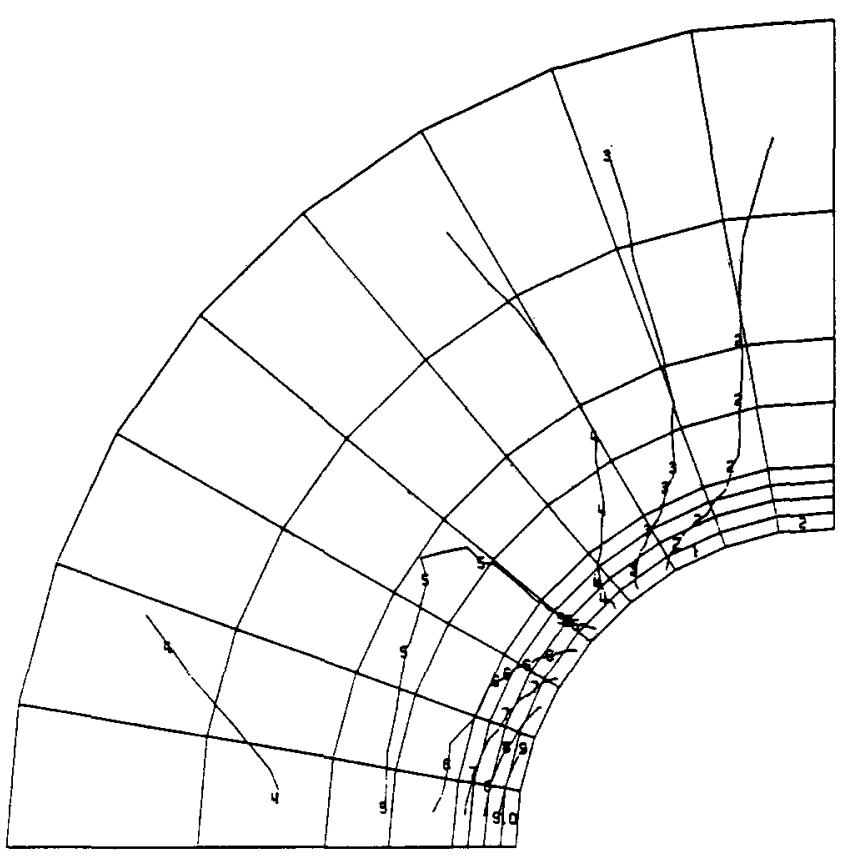

C

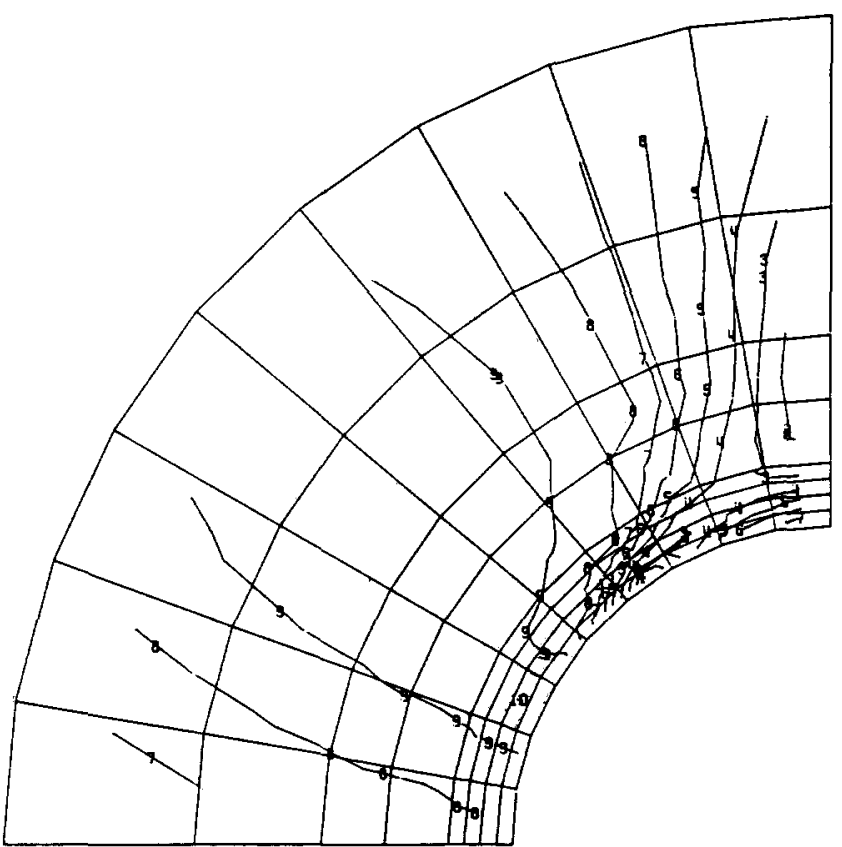

b

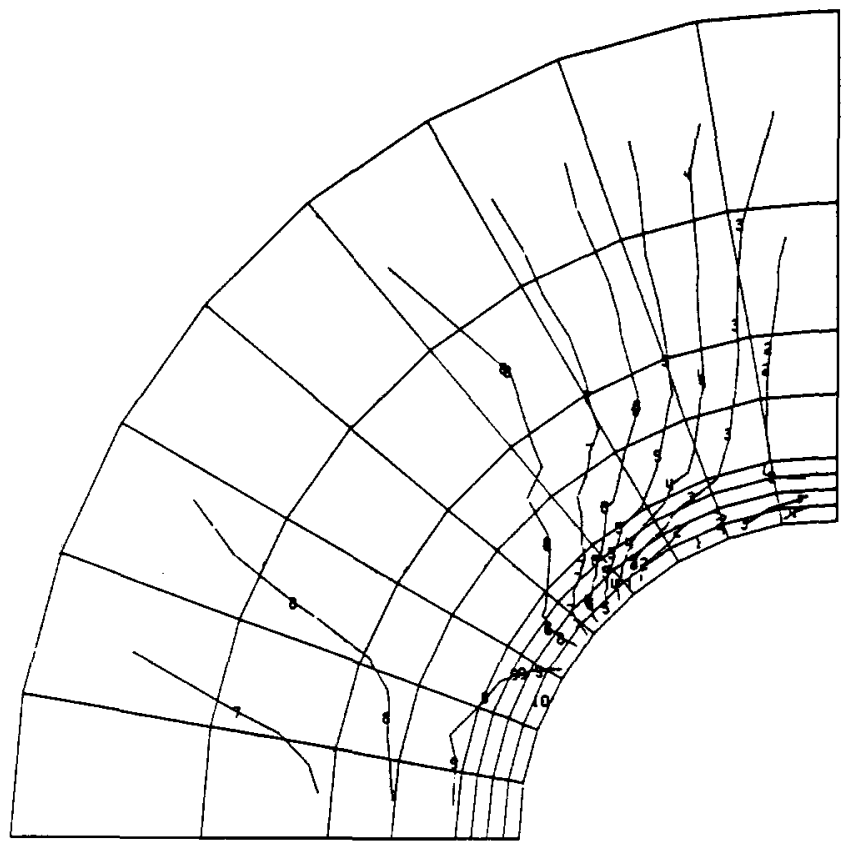

d

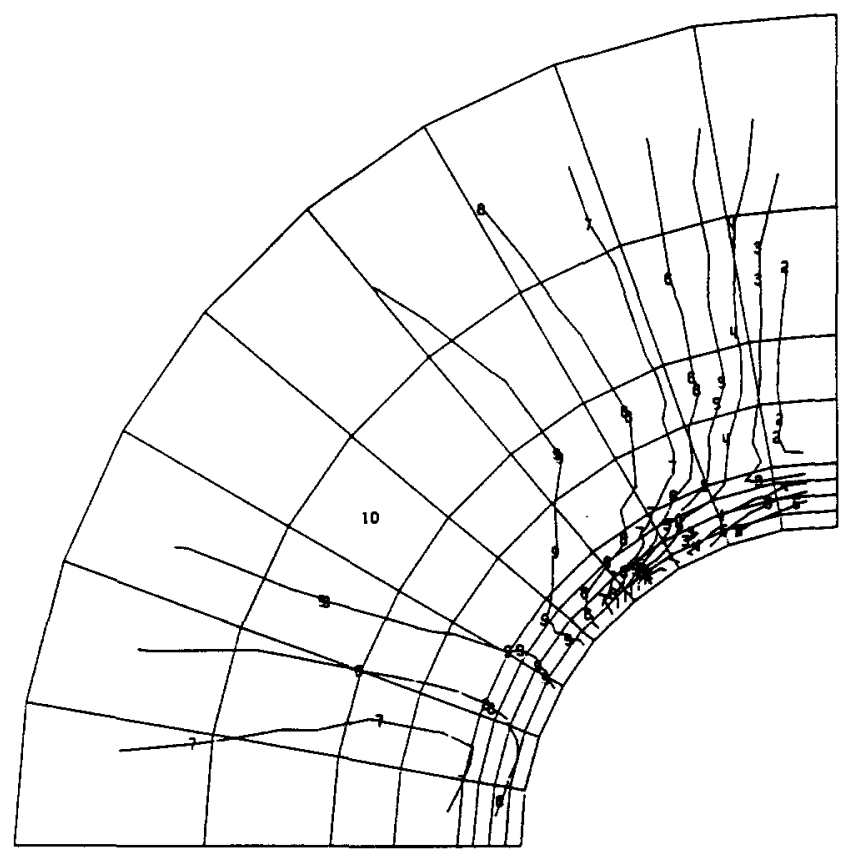

Figure 4 Typical $\tau_{\text {oct }}$ contour plots of the FEM results. In the case of PC/hole system, the maximum $\tau_{\text {oct }}$ (curve number 10 ) is located at the equatorial region when the stress state is still in the linear elastic regime, as shown in (a). When the material has a local stress state which exceeds the yield stress, the maximum $\tau_{\text {oct }}$ zone begins to shift upward and grow, as shown in (b) and (c). If the load is further increased, the available $\tau_{\text {oct }}$ zone grows to a preferred orientation (upward and outward), as shown in (d). Note that only part of the mesh is shown

corresponding region. The onset of shear yielding of the $P C$ matrix occurred at the equatorial region around the hole (Figure $4 a$ ) when the stress locally exceeded the yield stress of PC. If the load continued to increase after this point, then the maximum $\tau_{\text {oct }}$ would shift toward the $\theta=45^{\circ}$ region along the interface (Figure $4 b$ and $c$ ). If the load is further increased, the maximum $\tau_{\text {oct }}$ will shift both upward and outward at ca. $\theta=40^{\circ}$ zone (Figure $4 d$ ). The extent of the shift in the maximum $\tau_{\text {oct }}$ is dependent on the stress concentration near the interface and the difference between the yielding strain and the strain at which hardening occurs. At this stage, stable inclined necks begin to form. Careful examination of these four $\tau_{\text {oct }}$ contour plots (i.e. Figure 4) reveals that at stages before the inclined necks form, the extent of the yielded zone is rather limited. When the yielded zone grows to the preferred orientation (Figure 4d), the inclined neck, which is a consequence of the coalescence of massive shear bands, begins to develop. These descriptions just given above can be verified using $\mathrm{PC} /$ hole experiments. 

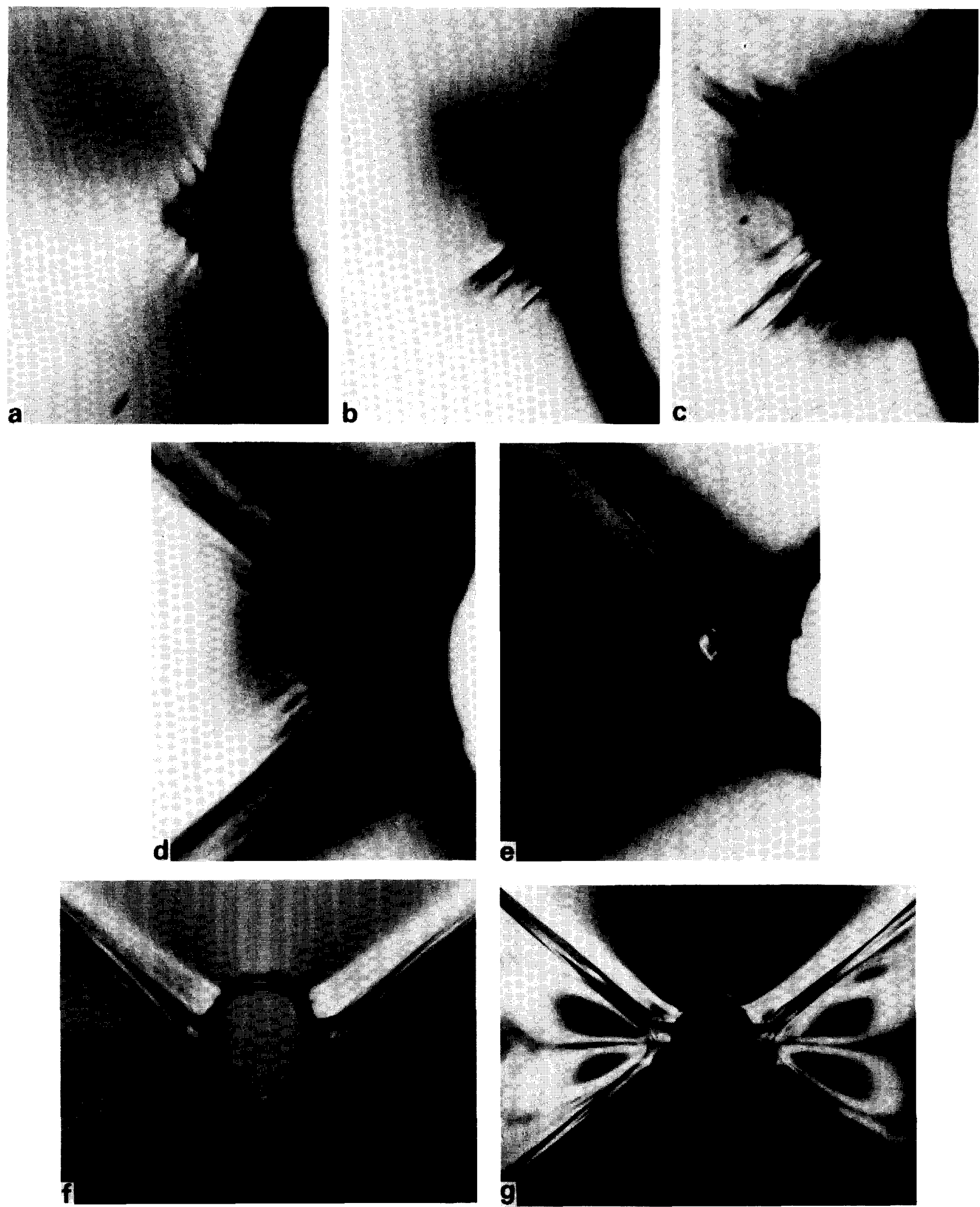

Figure 5 Tensile in situ optical micrographs of the PC/hole system ( $\frac{1}{4}$ " thick) under plane strain condition: (a) optical (bright field) micrograph taken at the onset of yielding of the PC at the equatorial region; (b)-(d) optical (bright field) micrographs taken progressively as the tensile load on the specimen is further increased; (e) optical (bright field) micrograph taken when the inclined neck finally forms, but prior to crack initiation at the equatorial region; (f)-(g) are optical micrographs taken, using bright field and cross polarized light, respectively, after unloading of the tensile specimen at stage (e). Note that the tensile direction is vertical and the hole size is $\frac{1}{16}$ " (the magnifications vary among these micrographs)

According to the classical closed-form solutions, the maximum $\tau_{\text {oct }}$ is located at $\theta=90^{\circ}$ (in the linear elastic range) at the interface between the more compliant inclusion (or hole) and the ductile matrix. Our FEM results in the low stress region agree with the classical closed-form solution. For the non-linear region, the observations made in the PC/hole experiments (Figure 5) are used to verify the FEM results. For clarity, only the 


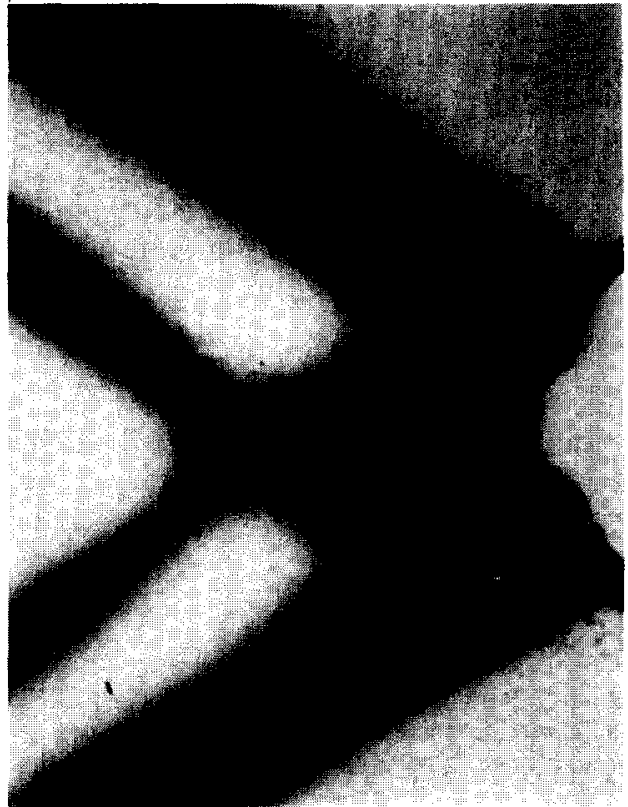

Figure 6 Optical micrograph of the plane-strain cracked specimen $\left(\frac{1}{4}{ }^{\prime \prime}\right.$ thick) of PC/hole system after unloading. As shown, the crack is initiated at the equatorial region and propagates in a direction perpendicular to the tensile direction. Note that the hole size is $\frac{1}{16} "$

plane strain case experiments are shown. Very fine shear bands did initiate at $\theta=90^{\circ}$ (Figure 5a) (onset of yielding). These plane strain shear bands cross each other in a manner describable as a slip-line field ${ }^{23}$. The slip lines grow along $\pm 40^{\circ}$ planes on either side of the equatorial plane. Subsequently, large diffuse shear zones above and below the initial slip lines formed and radiated away from the hole (Figure $5 b-d$ ). Eventually, inclined shear necks formed at $c a . \theta=40^{\circ}$ location at the interface (Figure $5 e$ $g$ ). These experimental observations demonstrate that, if the Von Mises yield criterion applies, the maximum $\tau_{\mathrm{oct}}$ shifts from $\theta=90$ to $\theta=45^{\circ}$ along the interface.

Comparison between the $\mathrm{PC} /$ hole experiments and the 2-D FEM experiment verifies that our simulation describes closely the $\sigma-\varepsilon$ field around the cylindrical inclusion (or hole) embedded in a continuous medium, under the conditions approximating plane stress (when a $\frac{1}{16}$ " thick specimen is used) and plane strain (when a $\frac{1}{4} "$ thick specimen is used). Experimental results obtained by Narisawa and coworkers ${ }^{23,24}$ are also consistent with both our experimental observations and computer simulations. The simulation work with $\mathrm{PC} /$ rubber, polyetherimide/PC, $\mathrm{PC} /$ polyethylene and epoxy/rubber, to be published in a separate paper, indicates that in many ways these systems are similar to the $\mathrm{PC} /$ hole case.

The PC/hole results may also be used to interpret some seemingly contradictory experiments ${ }^{25,26}$. In these experiments the shear bands were formed at a location away from the equatorial region. According to both the Tresca and Von Mises yield criteria, the microscopic shear bands should form at the equatorial region where the stress concentration is the highest. This apparent contradiction can be explained using our present findings. Since those experiments ${ }^{25,26}$ were performed under simple tension conditions, the maximum $\tau_{\text {oct }}$ is located at the equatorial region when the inclusion phase is rubbery and the deformation is in the linear region. According to our simulation, when the local stress near the interface exceeds the yield stress, the maximum $\tau_{\text {oct }}$ begins to shift upward. As a result, if the Tresca and Von Mises criteria are valid, the observed shear bands can be located at regions other than at the equator. This tentative explanation may be verified later using 3-D FEM work as well as experiments on epoxy/plastic ball model systems.

The good correlation between the FEM simulation results and the $\mathrm{PC} /$ hole experiments indicates that the numerical approach is applicable to the large scale deformation of polymers, even though shear bands are formed. The physical basis for the shift of the maximum $\tau_{\text {oct }}$ is probably due to an unloading effect of the polymer: when the stress exceeds the local yield stress due to the strain softening of the polymer, the yielded material element will transfer the load to the surrounding material which will, in turn, cause a shift in the location of the maximum $\tau_{\text {oct. }}$ (We note that even without strain softening the yield zone will spread beyond the equatorial region.) Furthermore, when the $\mathrm{PC} /$ hole specimen is tested to fracture, it is found that a pair of cracks initially are formed at the equatorial region of the hole. These cracks then propagate perpendicular to the tensile direction and fracturing occurs (Figure 6). This behaviour, in fact, is predicted by FEM. In the simulation, the maximum $\tau_{\text {oct }}$ shifts back to the equatorial region after reaching the $40^{\circ}$ region at the interface (Figure 7 ). The reverse shift in maximum $\tau_{\text {oct }}$ implies that the equatorial region begins to undergo strain hardening, which causes local fracturing of the material. Figure 8 summarizes how the maximum $\tau_{\text {oct }}$ shifts before crack forms. The sequence of the shift in maximum $\tau_{\mathrm{oct}}$ is labelled using consecutive numbers.

It is worth noting that the strain concentration, in fact, is always located at the equator even after a crack forms. Thus, the entire FEM simulation process suggests that

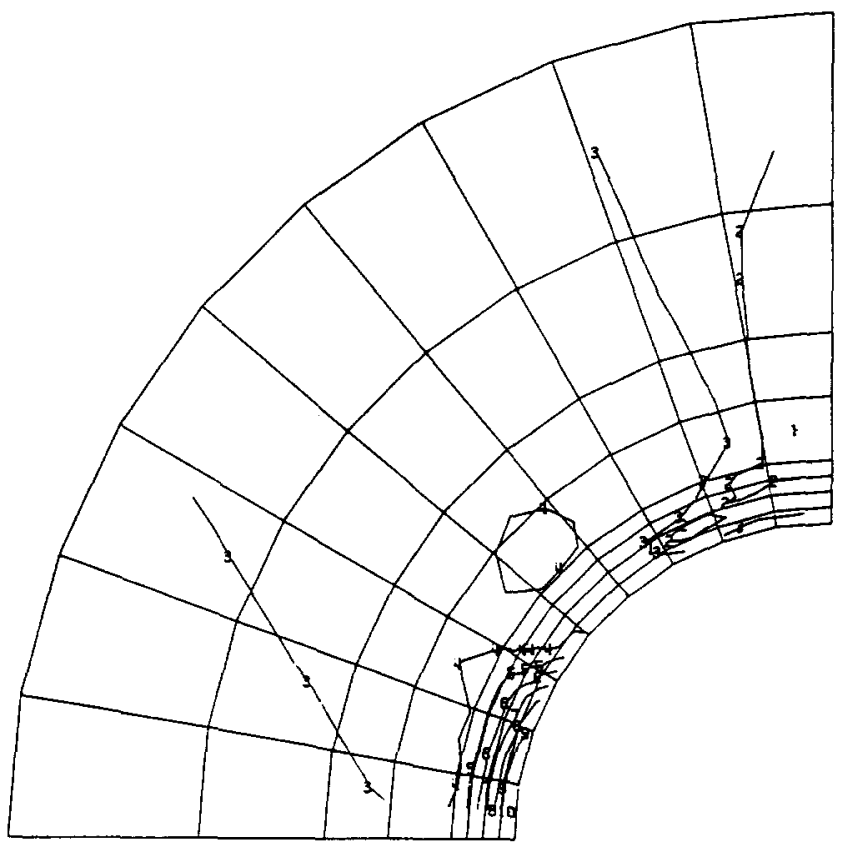

Figure 7 A $\tau_{\mathrm{oct}}$ contour plot of the FEM result. This plot shows the reverse shift of the maximum $\tau_{\text {oct }}$ (curve number 10 ) from the ca. $\theta=40^{\circ}$ region (see $F$ igure $4 d$ ) back to the equatorial region. The reverse shift in maximum $\tau_{\mathrm{oct}}$ implies that the equatorial region begins to undergo strain hardening, which causes local fracturing of the material. Note that only part of the mesh is shown 


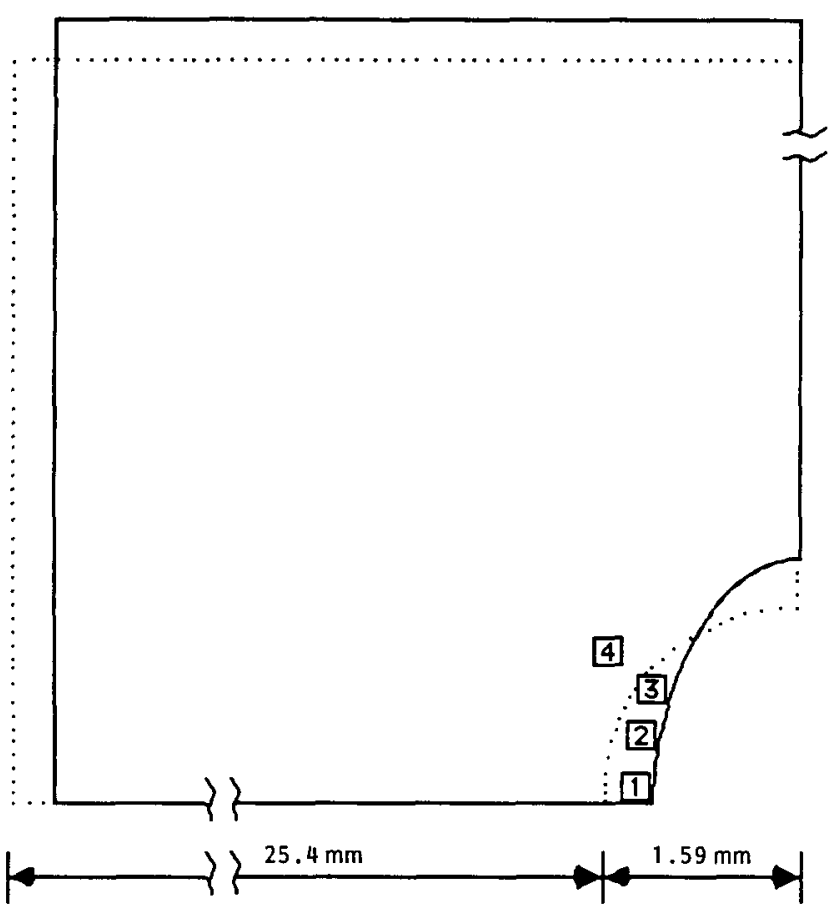

Figure 8 Summary of the shifts in maximum $\tau_{\text {oct }}$ from the FEM simulation results. The sequence of the shift is labelled with the consecutive numbers. In the elastic region, the maximum $\tau_{\text {oct }}$ is located at equator (i.e. (1)); when the material at the equatorial region yields, the maximum $\tau_{\text {oct }}$ shifts upward (i.e. (2)); if the system is further stretched, the maximum $\tau_{\text {oct }}$ shifts both upward and outward (i.e. (3) and (4)); at this stage if the strain at the equator exceeds the hardening strain, the maximum $\tau_{\text {oct }}$ will shift back to the equator (i.e. (1)), as a result, crack eventually forms. ——, Deformed and $\cdots \cdots$, undeformed shape

the deformation behaviour of ductile polymers is a straincontrolled process: in the elastic and anelastic regimes, the maximum $\tau_{\text {oct }}$ remains at the equatorial region; when yielding and strain softening occur at the equatorial region, the maximum $\tau_{\text {oct }}$ begins to shift towards $45^{\circ}$ angle near the interface between the two phases (unloading effect); as strain hardening begins to operate at the equatorial region, the maximum $\tau_{\text {oct }}$ shifts back to the equatorial region; not until the strain state reaches the fracturing point does the crack initiate and then propagate. At this point, the sample fails.

\section{CONCLUSION}

Two-dimensional FEM simulations, which involve consideration of the non-linear behaviour of a material, have been conducted on some two-phase polymer alloy systems. The PC/hole system was used to experimentally verify the most notable finding of the present simulation, namely, the shift of the maximum $\tau_{\text {oct }}$ from the $\theta=90^{\circ}$ to the $\theta=45^{\circ}$ region along the interface of the inclusion. This shift in maximum $\tau_{\text {oct }}$ can be interpreted as the unloading effect (strain softening) of the polymer, which locally exceeds the yield stress and transfers the load to the immediate surroundings. The entire deformation process is governed by the microscopic strain state of the polymer.

\section{ACKNOWLEDGEMENTS}

The work was partially supported by the National Science Foundation (Grant No. DMR-8708405), the E. I. DuPont de Nemours and Company, and the Macromolecular Research Center of the University of Michigan.

\section{REFERENCES}

1 Yee, A. F., Parker, D. S., Sue, H. J. and Huang, I-C. 'Toughening mechanisms in some rubber/plastic and plastic/plastic multiphase blends', PMSE Division Preprints, 194th American Chemical Society National Meeting, 1987

2 Koo, K., Inoue, T. and Miyasaka, K. Polym. Eng. Sci. 1985, 25, 741

3 Kurauchi, T. and Ohta, T. J. Mater. Sci. 1984, 19, 1699

4 Sue, H.J. and Yee, A. F. 'Toughening Mechamism(s) In Alloys of Rigid Polymers', RETEC (Chicago), The Society of Plastics Engineering, Chicago, 1987

5 Dekkers, M. E. J. PhD Thesis, Eindhoven University of Technology, The Netherlands, 1985

6 Bowden, P. B. in 'The Physics of Glassy Polymers' (Ed. R. Haward) Ch. 5, Applied Science Publishers, London, 1973

7 Robertson, R. E. Appl. Polym. Symp. 1968, 7, 201

8 Argon, A. S. and Bessonov, M. I. Phil. Mag. 1977, 36, 917

9 Brady, T. and Yeh, G. J. Macromol. Sci. (Phys.) 1974, B9 (4), 659

10 Rendell, R., Ngai, K. L., Fong, G., Yee, A. F. and Bankert, R. Polym. Eng. Sci. 1987, 27, 2

11 Haward, R. Polymer 1987, 28, 1485

12 Haward, R. (Ed.) in 'The Physics of Glassy Polymers', Ch. 6, Applied Science Publishers, London, 1973

Nimmer, R. Polym. Eng. Sci. 1987, 27, 16

Goodier, J. N. J. Appl. Mech. 1933, 1, 39

Dewey, J. J. Appl. Phys. 1947, 18, 578

Edwards, R. H. J. Appl. Mech. 1951, 18, 19

Eshelby, J. D. Proc. R. Soc. (London) 1957, A241, 376

Hashin, Z. J. Appl. Mech. 1962, 29, 143

Bevan, L. J. Polym. Sci., Polym. Phys. Edn. 1981, 19, 1759

Broutman, L. J. and Panizza, G. Int. J. Polym. Mater. 1971, 1,95 Agarwal, B. D. and Broutman, L. J. Fiber Sci. Technol. 1974, 7, 63

22 Ricco, T., Pavan, A. and Danusso, F. Polym. Eng. Sci. 1978, 18, 774

23 Narisawa, I., Ishikawa, M. and Ogawa, H. J. Mater. Sci. 1980, 15, 2059

24 Ishikawa, M., Narisawa, I. and Ogawa, H. Polym. J. 1976, 8, 391

25 Bucknall, C. B., Clayton, D. and Keast, W. E. J. Mater. Sci. $1972,7,1443$

26 Sternstein, S. S. in 'Treatise on Materials Science and Technology', Vol. 10 (Ed. J. M. Schultz) Academic Press, New York, 1977 